\title{
WAVELET-BASED POISSON SOLVER FOR USE IN PARTICLE-IN-CELL SIMULATIONS*
}

\author{
B. Terzić ${ }^{\dagger}$, D. Mihalcea, C. L. Bohn, Northern Illinois University, DeKalb, IL 60115, USA \\ I. V. Pogorelov, Lawrence Berkeley National Laboratory, Berkeley, CA 94720, USA
}

Abstract

We report on a successful implementation of a waveletbased Poisson solver for use in 3D particle-in-cell (PIC) simulations. One new aspect of our algorithm is its ability to treat the general (inhomogeneous) Dirichlet boundary conditions (BCs). The solver harnesses advantages afforded by the wavelet formulation, such as sparsity of operators and data sets, existence of effective preconditioners, and the ability simultaneously to remove numerical noise and further compress relevant data sets. Having tested our method as a stand-alone solver on two model problems, we merged it into IMPACT-T to obtain a fully functional serial PIC code. We present and discuss preliminary results of application of the new code to the modelling of the Fermilab/NICADD and AES/JLab photoinjectors.

\section{INTRODUCTION AND MOTIVATION}

PIC simulations are widely used in large-scale computational modeling in application fields as diverse as accelerator physics, galactic dynamics, plasma physics, and solid state device design. The PIC method [1] models the multiparticle dynamics of the system by tracking a set of tracer particles that sample the initial phase-space distribution function of the system. In quasi-static approximation, where the dynamics is modeled as the solution of the Vlasov-Poisson system of equations, the efficiency of the self-consistent inter-particle force calculation can be increased significantly by depositing the charge/mass distribution onto a computational grid, and solving the field equation on the grid. In accelerator physics applications, it is important that the on-grid Poisson solver used in PIC modeling be able to satisfy the following requirements:

1. account for multiscale dynamics, because even the fluctuations on smallest scales can lead to global instabilities and fine-scale structure formation, as exemplified by halo formation and microbunching instability observed in beam dynamics experiments [2,3],

2. minimize the numerical noise due to the fact that the number of particles used to sample the phase-space distribution function in the $N$-body simulation is several orders of magnitude smaller than the number of particles in the physical system which is being modeled, and

\footnotetext{
${ }^{*}$ Work of B.T., D.M. and C.L.B. is supported by Air Force contract FA9471-040C-0199. Work of I.V.P. is supported by the U.S. Department of Energy contract DE-AC03-76SF00098.

$\dagger$ bterzic@ nicadd.niu.edu
}

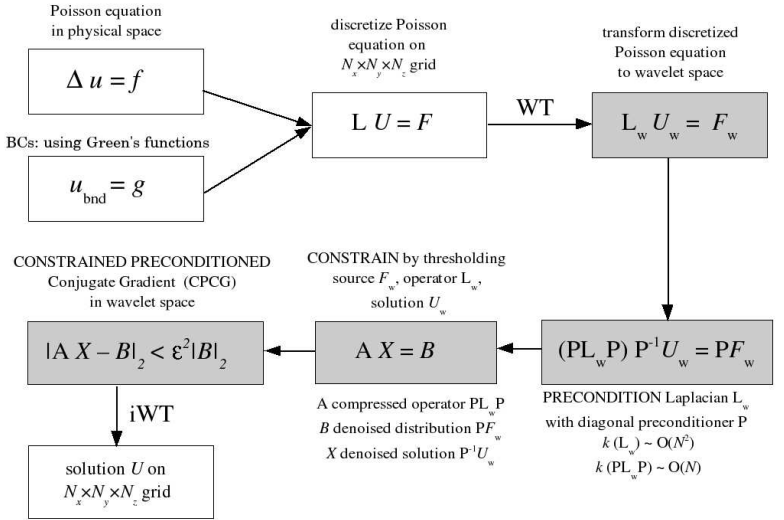

Figure 1: Flow-chart outline of the wavelet-based Poisson solver using (constrained) PCG method. The gray boxes represent the wavelet space; the physical space is in white. Constraining the PCG method is applied in the bottom middle box. The current version of the code does not have this step implemented yet.

3. be as efficient as possible in terms of computational speed and storage requirement, without compromising accuracy.

\section{DESCRIPTION OF THE SOLVER}

We have built on earlier work of Beylkin and co-workers $[4,5,6]$ to design and implement an iterative algorithm for solving the Poisson equation in three dimensions on the grid, subject to general (inhomogeneous) Dirichlet boundary conditions. A combination of three circumstances makes iterative solver particularly attractive in the current setting: Firstly, the (Laplacian) operator remains sparse in a wide variety of wavelet bases. Secondly, preconditioners for the Laplacian exist that are effectively diagonal in a wavelet basis. Finally, the fact that the Poisson equation has to be solved repeatedly, with the source term not changing significantly from one timestep to the next, simplifies to some extent the choice of the initial approximation (one can, e.g., use the potential computed on the previous timestep as the initial approximation for the presenttime potential).

As can be seen from the flow-chart in Figure 1, one begins by introducing a rectangular computational grid that envelopes tightly the density distribution, and whose boundaries may or may not coincide with the physical boundaries of the system on which the boundary condi- 
tions are specified. After computing the potential on the surface of the computational grid, one performs a Fast Discrete Wavelet Transform (FDWT) [7] on the source term and the Laplacian operator, solves the Poisson equation in the wavelet space, and performs the inverse FDWT to recover the on-grid potential in the physical (configuration) space. We now proceed to describe the boundary condition implementation in one important special case, and present the basic details of the preconditioned conjugate gradient (PCG) algorithm that forms the core of our solver.

\section{Implementation of Boundary Conditions}

A (Dirichlet) boundary condition often encountered in practice with beam dynamics simulations is that of the zero potential on the walls of an infinite pipe of rectangular cross-section, with open BCs in the longitudinal $(z)$ direction. The Green's function for such BCs is analytically known, so that the corresponding potential can be found by solving the set of equations

$$
\begin{gathered}
\rho^{l m}(z)=\frac{4}{a b} \int_{0}^{a} \int_{0}^{b} \rho(x, y, z) \sin \left(\alpha_{l} x\right) \sin \left(\beta_{m} y\right) d y d x, \\
\frac{\partial^{2} \phi^{l m}(z)}{\partial z^{2}}-\gamma_{l m}^{2} \phi^{l m}(z)=-\frac{\rho^{l m}(z)}{\epsilon_{0}} \\
\phi(x, y, z)=\sum_{l=1}^{N_{x}} \sum_{l=1}^{N_{x}} \phi^{l m}(z) \sin \left(\alpha_{l} x\right) \sin \left(\beta_{m} y\right),
\end{gathered}
$$

where $\rho$ is the charge distribution, $\phi$ is the potential, $\alpha_{l}=$ $l \pi / a, \beta_{m}=m \pi / b, \gamma_{l m}^{2}=\alpha_{l}^{2}+\beta_{m}^{2}, \epsilon_{0}$ is the permittivity of vacuum and the geometry of the pipe is given by $0 \leq$ $x \leq a$ and $0 \leq y \leq b[8,9]$. Eq. (3) is evaluated only on the surface of the computational grid, and for the predefined number of expansion coefficients $N_{x}$ and $N_{y}$.

\section{Preconditioned Conjugate Gradient}

The 3D Laplacian is a sparse, positive definite operator. The same holds true of the wavelet-decomposed Laplacian, assuming that one applies to it a thresholding procedure whereby all coefficients with magnitudes below a user-specified threshold are set equal to zero. In this setting, a conjugate gradient (CG) algorithm [10] provides an efficient way to invert the operator, the convergence rate depending on the condition number $\kappa[10]$ as

$$
\left|U-U^{i}\right|_{2} \leq\left(\frac{\sqrt{\kappa}-1}{\sqrt{\kappa}+1}\right)^{i}|U|_{2} .
$$

A major advantage of working in a wavelet basis is the existence of a diagonal preconditioner (first reported in [4] for the case of periodic BCs) that lowers $\kappa$ from $O\left(N^{2}\right)$ to $O(N)$. Our preconditioned conjugate gradient (PCG) algorithm is a combination of the operator formulation of the CG method with a wavelet-basis preconditioner.
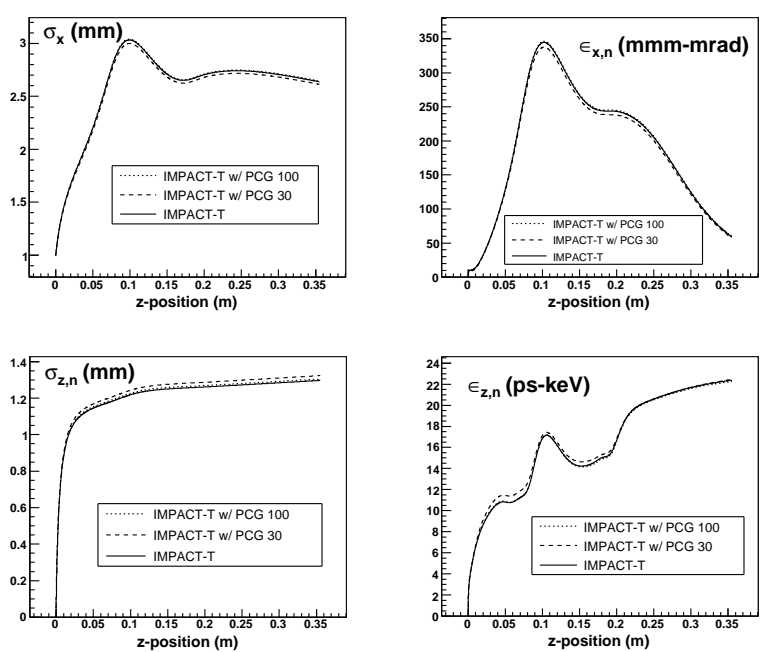

Figure 2: Comparison of IMPACT-T (solid lines), IMPACT-T with PCG and $N_{x}=N_{y}=30$ expansion coefficients (dashed lines) and IMPACT-T with PCG and with $N_{x}=N_{y}=100$ expansion coefficients (dotted lines) for the Fermilab/NICADD photoinjector (bunch charge of $1 \mathrm{nC})$.
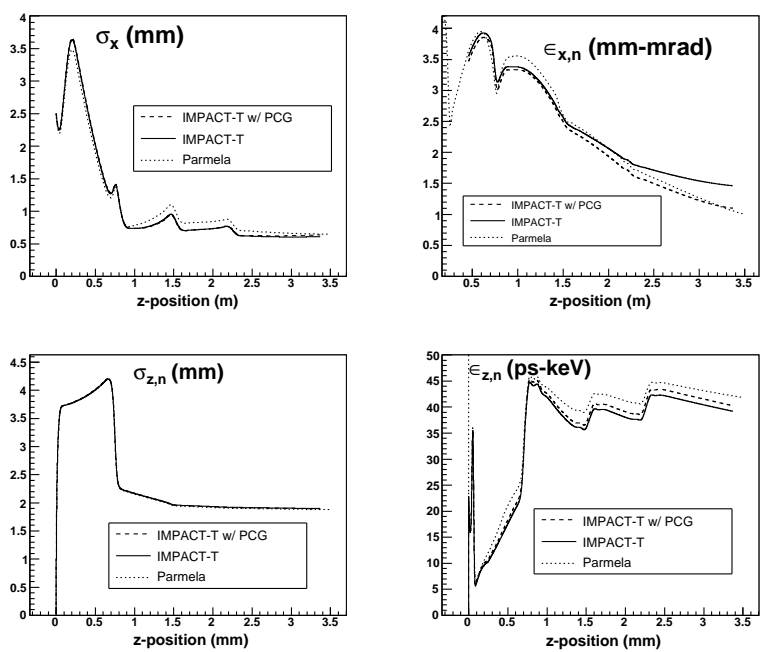

Figure 3: Comparison of IMPACT-T (solid lines), IMPACT-T with PCG and $N_{x}=N_{y}=30$ expansion coefficients (dashed lines) and PARMELA for the AES/JLab low-charge photoinjector (bunch charge of $133 \mathrm{pC}$ ).

Finally, we note that the thresholding operation performed both on operators and potential/density data sets is the simplest possible way to effect simultaneous compression and denoising. We are currently working on combining sparse array storage and manipulation algorithms with advanced, adaptive techniques for simultaneous compression and denoising to achieve new multiscale, highefficiency, high-fidelity PIC simulation capabilities. 

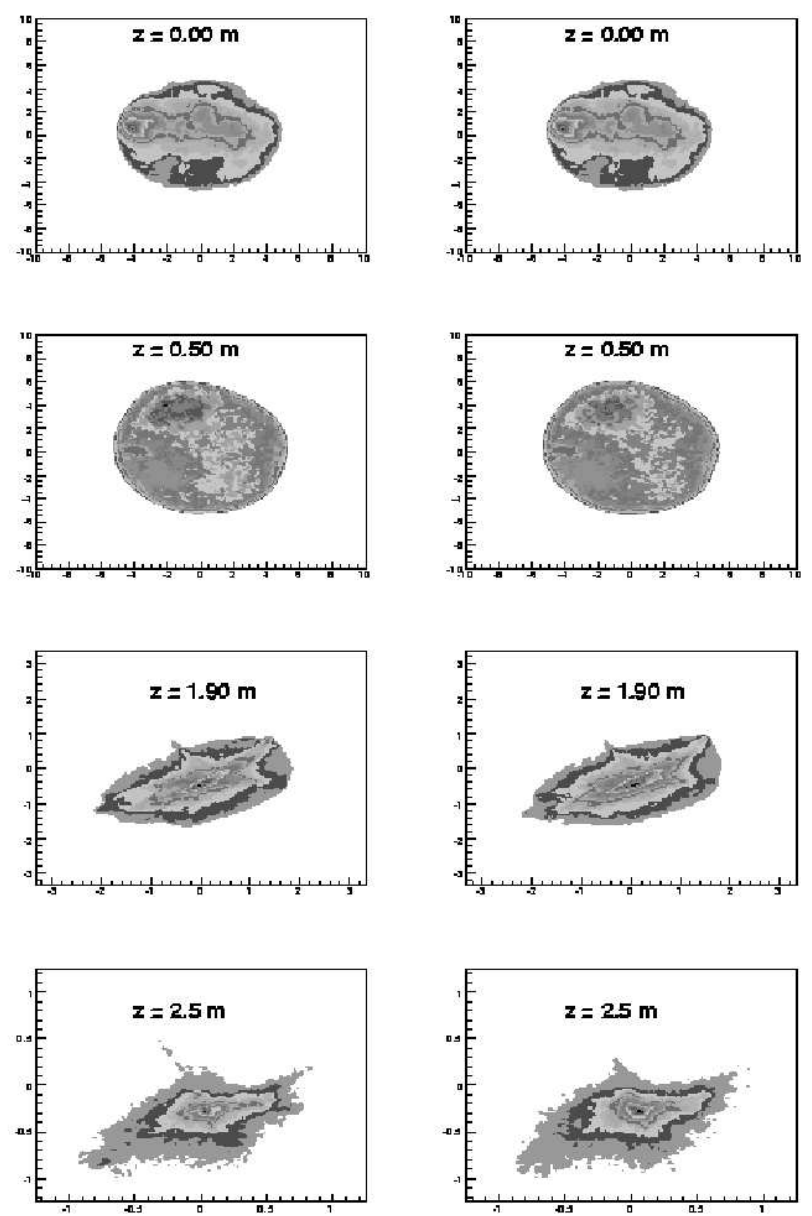

Figure 4: Comparison of IMPACT-T (left column) and IMPACT-T with PCG (right column) for transverse distributions at different $z$-locations along the AES/JLab lowcharge photoinjector (bunch charge of $133 \mathrm{pC}$ ).

\section{PHOTOINJECTOR SIMULATIONS}

Having tested the solver in the stand-alone mode on two three-dimensional test density distributions, one mimicking a charged particle beam and the other a galaxy [12], we have merged it into the IMPACT-T [8, 9] PIC code (replacing IMPACT-T's standard Poisson solver based on the use of Green's functions and the FFTs). Figures 2 and 3 present the results of simulations performed using IMPACT-T with the "native" solver and IMPACT-T with the new PCG solver. Figure 2 shows a standard set of rms diagnostics computed in a 200000-particle simulation of the Fermilab/NICADD photoinjector when electron transverse distribution is highly non-uniform. In this case, only true 3D algorithms, such as the one we discuss here, should be used. Figure 3 shows the same set of rms diagnostics for the AES/JLab photoinjector [11] for a uniform transverse distribution. The agreement among the results is clearly quite good. Going beyond the rms characterization of dynamics, Figure 4 illustrates the level of agreement in resolving the fine-scale structure of the charge den- sity distribution; the latter was computed in simulations of the AES/JLab photoinjector performed with 200000 simulation particles with non-uniform transverse initial distribution. As for the computational efficiency of the new solver, in serial simulations, runtimes for the two versions of IMPACT-T are within $10 \%$ of each other (the exact ratio depending on the total simulation length). Implementation of the denoising and compression will lead to further improvements in performance.

\section{CONCLUSIONS}

A new, wavelet-based 3D solver for the Poisson equation subject to general (inhomogeneous) Dirichlet boundary conditions has been implemented and successfully integrated into IMPACT-T. The solver is based on the operator formulation of the preconditioned conjugate gradient method. It takes full advantage of sparsity of waveletdecomposed operators and data sets, existence of efficient (diagonal) preconditioners in wavelet bases, and recursive nature of the PIC method. The new solver has been tested using standard IMPACT-T simulation results as a benchmark, and subsequently used in modeling the Fermilab/NICADD and AES/JLab photoinjectors. To our knowledge, work reported here and in [12] constitutes the first application of the wavelet-based multiscale methodology to beam dynamics simulations.

\section{REFERENCES}

[1] R. Hockney and J. Eastwood, "Computer Simulations Using Particles", Institute of Physics Publising, London (1988).

[2] C. L. Bohn and I. V. Sideris, Phys. Rev. Lett., 89 (2003), 214802.

[3] J. Qiang, R. D. Ryne and I. Hofmann, Phys. Rev. Lett., 92 (2004), 174801.

[4] G. Beylkin, In "Wavelets: Mathematics and Applications", eds. J. Benedetto and M. Frazier, CRC Press LLC, Boca Raton, Florida, 1993.

[5] A. Averbuch, G. Beylkin, R. Coifman, P. Fischer and M. Israeli, http://www.cs.tau.ac.il/ amir1/PS/poisson.pdf

[6] A. Averbuch, G. Beylkin, R. Coifman, P. Fischer, M. Israeli, In "Signal and Image Representation in Combined Spaces", eds. Y. Zeevi and R. Coifman, Academic Press, San Diego, 1998.

[7] I. Daubechies, "Ten Lectures on Wavelets", SIAM, Philadelphia, 1992.

[8] J. Qiang, R. D. Ryne, S. Habib and V. Decyk, J. Comp. Phys., 163 (2000), 434.

[9] J. Qiang and R. D. Ryne, Comp. Phys. Comm., 138 (2001), 18.

[10] G. H. Golub and C. F. Van Loan, "Matrix Computation", The Johns Hopkins Press, Baltimore and London (1996).

[11] A. Todd et al., In Proc. 2003 Part. Accel. Conf., IEEE Cat. No. 03CH37423, 977 (2003).

[12] B. Terzić and I. V. Pogorelov, Ann. of New York Acad. of Sci., 1045 (2005), in press. 